\title{
Du français sur objectifs spécifiques pour les professionnels du tourisme
}

\author{
Paula Santander Sotomayor ${ }^{7}$
}

Universidad Nacional, Costa Rica

\section{RESUMEN}

En este artículo se analiza la experiencia de un proyecto para la elaboración de cuatro módulos de francés para guías turísticos, los diferentes elementos que se incluyen para este fin y su posible adaptación a la metodología de «Vacances Cubaines» en el contexto costarricense.

\section{Résumé}

Dans cet article, on analyse l'expérience d'un projet de construction de quatre modules de français pour des guides touristiques, les différents éléments qui entrent en jeu et son adaptation à la méthodologie de « Vacances Cubaines » dans un contexte costaricien.

Palabras clave: francés del turismo, enseñanza del francés, FLE, módulos para guías del turismo.

Mots-clés : français du tourisme, enseignement du français, FLE, modules pour guides touristiques.

Le Costa Rica présente une réalité attrayante dans le secteur touristique mais malgré les efforts de développement du tourisme, la

$\overline{1}$ Correo electrónico: paula.santander@hotmail.com 
globalisation exige une qualité du service et une formation touristique qui, actuellement, sont insuffisantes dans ce domaine et inexistantes en ce qui concerne le français du tourisme. Par ailleurs, le flux des touristes venant des États-Unis est conditionné par les événements politiques et économiques qui ont lieu dans ce pays, ceux-ci pouvant entraîner une baisse importante de visiteurs et donc en souffrir les conséquences.

C'est à partir de ce constat problématique que ce travail a pris un sens, face à la nécessité de diversifier ce flux touristique, face au besoin de répondre à une clientèle européenne, qui nous exige donc, de doter les professionnels de compétences de communication dans un besoin urgent de formation linguistique spécialisée.

Nous avons donc voulu construire un projet de formation en ce qui concerne les professionnels du tourisme au Costa Rica, afin de couvrir des besoins de formation et promouvoir le tourisme costaricien dans le domaine de la francophonie dans une perspective interculturelle.

\section{Les paramètres situationnels}

Avant de définir un contenu linguistique à enseigner, il faut tenir compte de divers paramètres de la situation dans laquelle cet enseignement se réalise. Pour cela, il est nécessaire de prendre un certain recul, afin d'observer de manière objective quel est le rôle de chacun des éléments impliqués dans cette situation pour mieux déterminer les contenus et essayer de prendre les décisions correctes par rapport aux besoins et demande des apprenants pour qu'il y ait un équilibre et une adéquation entre ce contenu et les objectifs que l'on cherche à atteindre. Il faut signaler que la détermination des objectifs et besoins doit également se faire en tenant compte de l'environnement et des différents agents qui interviennent dans la situation d'enseignement, et qui peuvent représenter une situation réelle n'étant pas toujours compatible avec les attentes des apprenants, de l'enseignant ou même des institutions. 


\section{Les institutions concernées}

L'idée de ce projet de formation est née d'un besoin et une demande sociale existante depuis longtemps au Costa Rica mais qui pour différentes raisons, n'a pas pu être captée auparavant, et qui semblait enfin être reconnue par différentes institutions intéressées. Le rôle qu'ont joué ces institutions a été primordial parce que leur appui financier et leur poids politique, représentaient le moyen qui servait à concrétiser, faciliter et formaliser les contacts nécessaires en vue de la mise en place d'une formation. Sans leur intervention et leur collaboration, il aurait été compliqué de mener à bien un programme de formation.

Les institutions qui ont participé dans ce projet de formation pour professionnels du tourisme ont été le Service Culturel de l'Ambassade de France, l'Alliance Française de San José, l'ICT et l'Association Costaricienne des Professionnels du Tourisme.

\section{La définition du public et ses besoins}

Devant une situation d'enseignement/apprentissage dans laquelle, apprenants, enseignant et institutions se trouvent impliqués, il est nécessaire de décrire et analyser les différents paramètres, en recueillant des informations sur ses acteurs. Il s' agissait alors de cerner le public et se demander qui était l'apprenant et quels étaient ses besoins. Toutefois, il faut signaler que ce type d'investigation doit se faire entre enseignant et apprenants, tout au long du parcours d'apprentissage et non seulement avant le cours, et qu'il s'agissait donc d'un premier aperçu qui ne pouvait pas à ce moment-là, présenter toutes ses caractéristiques.

\section{Le public}

Il s'agissait d'un public non captif, composé de 15 guides touristiques costariciens, qui souhaitaient apprendre le français avec l'intention de pouvoir l'utiliser dans leur pratique professionnelle. Ils montraient et exprimaient déjà avec grand enthousiasme leur intérêt 
d'apprendre et se sentaient privilégiés car ils savaient que d'autres guides auraient voulu y participer et que les facilités économiques qui leur avaient été données représentaient une occasion à ne pas manquer. Ils semblaient donc avoir des besoins et des motivations réels.

Les quinze guides avaient une parfaite connaissance de l'anglais et l'utilisaient quotidiennement dans leur pratique professionnelle. Cet élément risquait d'entraver l'enseignement/apprentissage au niveau de la prononciation de quelques apprenants car l'accent anglais semblait même l'emporter sur leur langue maternelle et influencer l'expression orale des apprenants. Cette situation ne représente aucun problème s'il s'agit d'un accent localisé, que les touristes apprécient souvent lorsqu'ils visitent un pays par son aspect exotique. En outre, l'influence de l'anglais en tant que langue « dominante » et universelle, et la pénétration culturelle des Etats-Unis n'épargne pas un petit pays comme le Costa Rica, proche géographiquement et fortement dépendant économiquement. On retrouve souvent cette situation chez les élèves qui apprennent le français. Même s'ils maîtrisent d'autres langues telles que l'allemand ou l'italien, c'est l'anglais qui se superpose et qui devient inconsciemment la langue de référence au moment de prononcer le français.

Cependant, les attitudes des apprenants vis-à-vis du français semblaient positives et non stéréotypées car ils avaient et ont l'habitude, dans leur pratique professionnelle, d'être en contact avec des touristes de nationalités différentes et sont conscients des différences culturelles face auxquelles ils tentent d'avoir une attitude compréhensive.

C'est surtout au niveau de la complexité que comporte la langue par rapport au fossé qui existe entre le français de l'oral et de l'écrit, qu'il semblait y avoir le plus d'insécurité de leur part, et face à la difficulté de mettre en relation ces deux compétences.

Un autre point important dont il a fallu tenir compte et qui était, sans aucun doute, le plus grave, c'était le temps dont ils disposaient pour leur apprentissage. En effet, le cours avait commencé en pleine 
saison haute, période dans laquelle les guides touristiques avaient le plus de demandes professionnelles et étaient le plus sollicités, car la présence de touristes à cette époque de l'année est la plus massive. C'est pour cette raison que le temps consacré à l'apprentissage, en classe et chez eux, semblait très limité, car cette période représentait en outre, les entrées économiques les plus importantes qui allaient également constituer leur réserve pour les mois à venir où les flux touristiques diminuent considérablement.

Même en étant tous conscients de ce problème, l'absentéisme risquait donc d'entraver la progression de l'enseignement/ apprentissage. Une autre caractéristique importante pour l'identification des besoins de formation, était que les guides touristiques devaient utiliser le français en situation orale, en face à face avec les touristes francophones, ayant très peu recours à l'écrit dans leur pratique professionnelle. Aucun d'entre eux n'a exprimé le besoin de faire un travail systématique sur l'expression et compréhension écrites car dans leurs activités professionnelles cet aspect était secondaire.

Le public avec lequel nous avons travaillé présentait des habitudes d'apprentissage diverses. Leurs stratégies d'apprentissage variaient d'un apprenant à l'autre. Certains avaient recours à une manipulation directe de la langue, à ce que Oxford appelle les stratégies mnémoniques, et révisaient leur cours régulièrement, d'autres utilisaient des techniques précises de manipulation, ils analysaient et raisonnaient en faisant des comparaisons avec leur langue maternelle ${ }^{2}$. Cependant, peu d'entre eux planifiaient et aménageaient leur apprentissage. Le fait de prendre en main ce qu'ils apprenaient avec une certaine indépendance, ne semblait pas être culturellement appris. En général, les apprenants étaient en majorité très dépendants de l'enseignant, ou des autres apprenants, ou de ce qui se passait en classe. Par manque de temps ou pour des raisons culturelles, il était peu

\footnotetext{
Rebecca Oxford, Language Learning Strategies: What Every Teacher Should Know' (New York : Newbury House, 1990) 137-139.
} 
fréquent qu'ils veuillent gérer eux-mêmes, une partie de leur apprentissage ou du moins, on ne leur avait jamais appris à le faire.

Mais ce qui était très marqué dans ce groupe de guides touristiques, c'est qu'ils avaient recours surtout à ce que Oxford appelle, des stratégies indirectes, affectives et sociales. Ils posaient des questions, coopéraient avec les autres, s'auto encourageaient, encourageaient les autres et cultivaient l'empathie.

\section{Ses besoins}

L'identification des besoins est liée à divers concepts tels que la notion d'attente, de demande, de motivation, d'objectif, de ressources et autres encore. L'analyse des besoins a suscité de nombreux commentaires et critiques des modèles d' analyse proposés par René Richterich ou encore par John Munby. Lehmann ${ }^{3}$ reprend l'idée de Louis Porcher selon laquelle l'analyse des besoins est en même temps indispensable et dangereuse Indispensable car elle est liée à la définition des objectifs de tout apprentissage et dangereuse car souvent cette analyse se fait sans consultation du public. Le modèle de Richterich était considéré comme extrêmement lourd mais aujourd'hui, il fait référence à la nécessité de chercher des pratiques plus légères où il s'agit de tenir compte des particularismes de la situation d'apprentissage et des apprenants, en considérant, non seulement les besoins langagiers mais également les aspects socioculturels, les habitudes d'apprentissage des apprenants et leur représentation linguistique. Ce n'est plus alors la langue en tant qu'objet d'enseignement qui est au centre, mais l'apprenant, avec son environnement social qui est pris en compte, en vue de le satisfaire du mieux possible, le rendre responsable et acteur de sa formation et surtout conscient de ses besoins.

Déterminer les besoins revient également à déterminer les besoins d'apprentissage, qui traduits en actes de communication, représentent

${ }^{3}$ Denis Lehmann, Objectifs spécifiques en langues étrangères (Paris : Hachette, 1993). 
l'ensemble de ce qui est nécessaire d'apprendre ou de savoir faire pour apprendre efficacement à communiquer, notamment dans les situations cibles et considérer ce que les apprenants estiment qu'ils doivent être en mesure de faire et ce qu'ils peuvent faire dans un contexte d'enseignement/apprentissage déterminé.

Il faut que cette analyse se fasse de façon permanente car l'apprentissage est en constante évolution et les besoins initiaux ne représentent pas tous les besoins qui apparaissent au cours de l'apprentissage.

Il faut réunir les besoins langagiers et nonlangagiers et tenir compte des besoins d'apprentissage et ses composantes psychoaffective et socioculturelle pouvant également être cernées dans l'étude des situations cibles.

En ce qui concerne les besoins langagiers qui mettent en œuvre un type de discours spécifique selon les situations de communication, il est difficile de prévoir à l'avance, les échanges langagiers en tant que tels, mais on peut toutefois s'en faire une idée. Pour cela, il est nécessaire de faire l'inventaire des situations d'échanges langagiers car elles constituent un outil de repérage de l'identification des besoins. Il est nécessaire d'analyser les échanges langagiers et actes de paroles qui se retrouvent dans ces situations et les raisons pour lesquelles se traduit l'échange ou l'activité tout en sachant que les échanges en classe varient de ceux qui ont lieu dans les situations professionnelles réelles.

Après avoir demandé aux apprenants d'établir en langue maternelle une liste générale des situations orales de communication dans lesquelles ils avaient besoin d'utiliser la langue, voici ce qui en a résulté :

- donner la bienvenue à un groupe de touristes

- $\quad$ se présenter, et présenter le chauffeur

- $\quad$ expliquer le programme (ce que les touristes vont faire durant le tour, sa durée)

- expliquer l' accès aux toilettes du bus 
- donner une explication générale sur les industries importantes au Costa Rica (banane, plantes, fleurs, bétail, café, noix)

- donner une explication générale au sujet de la société costaricienne (les classes sociales, les différents types d'impôts, le salaire minimum, le système de sécurité sociale et le système éducatif)

- décrire le climat (microclimats, humidité, temps, pluviosité)

- décrire le relief (volcans, plaques tectoniques, tremblements de terre)

- décrire la faune et la flore (les différentes espèces animales, végétales, les différents types de forêts)

A ceci, il faut ajouter, les besoins langagiers et nonlangagiers des guides de répondre à des plaintes, demandes, questions, commentaires des touristes ainsi qu'aux situations accidentelles devant lesquelles les touristes et les guides pourraient se retrouver.

Cependant, dans la réalité des choses, et ne pouvant pas tout aborder à la fois, toutes ces situations d'échanges langagiers ne pourraient être comprises dans une formation de 52 heures. Elles seraient bien sûr prises en compte dans la continuation de cette formation. Il a donc fallu négocier avec les apprenants afin de décider et sélectionner, celles qui paraîtraient les plus pertinentes pour cette première session, et qui nous serviraient d'outils pédagogiques pour déterminer des contenus langagiers et culturels en vue de communiquer.

En ce qui concerne les besoins psychoaffectifs des apprenants, il est important d'être constamment conscient de cet aspect et savoir qu'il est directement en relation avec l'environnement et le vécu de l'apprenant.

Les apprenants et surtout les débutants ont tendance à ressentir une certaine insécurité face à l'apprentissage et la satisfaction de ce besoin ne peut se faire qu'en informant la situation qui sera vécue dans la classe et en la rendant proche de sa réalité. Mais l'attitude de 
l'enseignant face à ce sentiment d'insécurité, et la souplesse et flexibilité qu'il emploie au niveau affectif envers l'apprenant et envers la représentation que l'apprenant se fait de la langue cible, sont importantes. Il faut que cette sensation de régression soit normalisée à l'aide de l'enseignant et que la représentation de la langue cible se comprenne, non pas en tant que langue dominante par rapport à la langue source mais, dans un effort interculturel de compréhension et ouverture.

C'est donc là, que la composante socioculturelle des besoins d'apprentissage, joue un rôle prépondérant. Car en effet, enseigner une culture c'est découvrir et prendre conscience de ce que nous sommes, c'est s'accepter et accepter l'autre dans leur culture correspondante, sans tomber dans les idées toutes faites, et cela doit se faire, dans un processus de construction mutuelle. C'est pourquoi l'enseignement/apprentissage, dans une perspective interculturelle est nécessaire pour les enseignants et les apprenants, car elle s'oppose aux conceptions traditionalistes et idéologiques de la culture et la civilisation, et enrichit ainsi notre vision du monde. Tenir compte de la composante socioculturelle inclut l'aptitude à établir un rapport avec autrui, une certaine connaissance de son mode de vie, une conscience de soi et de la façon dont les autres nous voient, une aptitude à découvrir et à entrer dans de nouvelles situations d'échange interculturel.

Défi tout de même, pour les enseignants ainsi que pour les apprenants, parce qu'on touche là, un point qui est toujours délicat et qui se lie à la composante psychoaffective, à savoir, notre monde intérieur, l'humain, et le vécu qui porte l'histoire qu'il y a en nous.

Ces composantes qui doivent être prises en compte dans les besoins d'apprentissage regroupent également l'importance du rythme individuel de l'apprenant, et de ses stratégies d'apprentissage. Pour cela, il serait nécessaire de permettre une participation active et dynamique dans la classe, au moyen de techniques qui provoquent des réactions, des questionnements, des remarques, des observations, afin de déclencher des interactions. 


\section{Un matériel de référence : «Vacances Cubaines »}

L'élaboration de « Vacances Cubaines » a commencé en 1993 à Cuba en vue d'appuyer et promouvoir le tourisme en tant qu'action économique prioritaire dans ce pays et dans le besoin de former des professionnels en français. Le travail a été réalisé par une équipe composée de spécialistes français du Centre de Recherches et d'Applications Pédagogiques Langues (CRAPEL) et de spécialistes cubains faisant partie de l'École de Hautes Études d'Hôtellerie et Tourisme (EAEHT).

Il s'agit de propositions pour une formation en français de spécialité qui contient une méthodologie pouvant être globalisée et dont l'organisation peut s'étendre à d'autres situations. Le principe fondateur est l'approche communicative et se base sur l'idée qu'on ne peut parler du français du tourisme sans savoir : qui ? À qui ? Pour qui ? et à quel contexte social, temporel, spatial on se réfère.

"Vacances Cubaines" se présente à travers des tâches professionnelles à réaliser, réunies en trois ensembles fonctionnels qui correspondent chacun à trois manuels : « Fonctions d'animation », "Relations avec la clientèle » et « Fonctions de service ». Une partie de ces tâches est caractérisée par les professions de service et se font à partir de l'idée que « le client est le roi », et donc, que ses différents critères culturels sont importants, ainsi que les représentations du pays qu'ont les professionnels et le rôle de la compétence interculturelle dans leur apprentissage. Une autre partie des tâches est caractérisée par les professions de contact, basée sur des éléments tels que la durée de ces contacts, leur qualité, leur quantité, leur importance, le rôle professionnel et l'influence de la personnalité dans ceux-ci.

"Vacances Cubaines » s' organise également autour d'objectifs spécifiques communicatifs qui comportent des thèmes, tient compte des aptitudes devant la tâche à réaliser et des objectifs linguistiques se référant au lexique, aux formulations, au registre, à la prononciation et la grammaire. 
Elle propose différents modules qui sont indépendants les uns des autres pouvant être ainsi travaillés selon les intérêts et besoins de l'apprenant ou selon les critères du groupe et de l'enseignant.

L'élément le plus attrayant de « Vacances Cubaines » est sans doute la grande dimension culturelle qu'elle a en elle, car elle contient des objectifs interculturels et présente des informations sur la France, les Français, les touristes français, leurs exigences et leurs caractéristiques socioculturelles, en espagnol, ce qui permet aux apprenants d'être en contact direct avec ces informations, sans obstacle linguistique. Les documents (authentiques, réalistes ou fabriqués), les consignes et les objectifs qui traitent cette information sont également présentés en espagnol.

Il faut remarquer, en ce qui nous concerne, que « Vacances Cubaines » ne comporte pas de progression mais des modules indépendants. Il s'agit également d'une méthode qui n'inclut pas tout ce dont on a besoin, mais d'où l'enseignant et (ou) l'apprenant peuvent extraire ce qui leur est nécessaire et déduire quels sont les points linguistiques que l'apprenant a besoin d'acquérir en utilisant, si nécessaire, d'autres ressources didactiques que les modules.

\section{Les exigences et possibilités d'adaptation}

C'est en nous basant et en nous inspirant d'une méthodologie et des objectifs, activités et tâches que propose « Vacances Cubaines », que nous avons essayé de construire la formation en question. Pour cela, il était nécessaire d'en faire une adaptation réaliste, qui nous permettrait de suivre le modèle méthodologique qu'elle nous proposait tout en l'intégrant en accord avec le contexte et la situation d'enseignement/apprentissage de cette formation.

L'adaptation que nous avons réalisée s'est définie et s'est basée sur quatre aspects essentiels. Le premier consistait à utiliser tels quels, les documents présentés en espagnol qui présentaient des informations culturelles sur les Français et les documents dont les apprenants se serviraient pour leur auto-apprentissage. 
Le deuxième, reprenait des thèmes qui informaient sur la culture cubaine pour les remplacer par des informations sur la culture costaricienne, et s'il était nécessaire, en ajouter d' autres qui pourraient être représentatifs du Costa Rica.

Le troisième aspect de l' adaptation consistait à centrer le travail sur l'organisation méthodologique de "Vacances Cubaines " présentée par des tâches professionnelles à réaliser, à travers des activités de compréhension orale, d'expression orale et ses rubriques «Se préparer », «S'exercer » et « Mettre en pratique ».

Pour cela, et c'est le quatrième point important, il fallait sélectionner les tâches professionnelles qui étaient aptes à notre public, au temps dont on disposait et à ses besoins dans leur pratique professionnelle. Tous les objectifs de "Vacances Cubaines» ne pouvaient évidemment pas être travaillés car ils devaient tout d'abord être localisés par rapport aux situations de communication déjà mentionnées auparavant et parce que les limitations de temps ne nous le permettaient pas.

Il faut dire que ce qui était le plus intéressant pour une adaptation, c'était les caractéristiques de la méthodologie en soi et la manière dont les activités et objectifs étaient proposés.

En effet, l'organisation d'un module est structurée de façon à pouvoir sélectionner facilement les objectifs dont nous avons besoin car chaque tâche professionnelle à réaliser, se présente de manière indépendante et s'insère dans un travail sur les quatre habiletés, en privilégiant fortement la compréhension et expression orales. Par conséquent, ce sont les objectifs et activités orales les plus proches des besoins des apprenants, que l'on a pu adapter au contexte.

Un deuxième aspect qui n'a posé aucun problème au moment de l'adaptation est l'élément culturel de "Vacances Cubaines». Tout le matériel écrit proposé dans sa dimension sociale peut être travaillé. En effet, de nombreux documents écrits sont en espagnol. Ceci facilite l'insertion du culturel et de l'interculturel dans la classe et en dehors d'elle, en donnant la possibilité aux apprenants de consulter et travailler 
ces documents chez eux en vue d'en discuter le contenu en classe par exemple.

Un troisième élément lié au deuxième est celui de l'autoapprentissage. Afin d' amener les apprenants à gérer leur apprentissage et apprendre à apprendre, « Vacances Cubaines » propose différentes activités et documents écrits qui facilitent ce travail. Vu que l'assistance aux cours semblait parfois être difficile à réaliser, il était indispensable que les apprenants aient l'opportunité de découvrir la possibilité d'élargir leurs connaissances linguistiques et culturelles grâce à des activités qui les aident à prendre conscience de l'existence de stratégies de cet apprentissage, et de cette manière, apprendre à les mettre en pratique.

Toutefois, la méthodologie de "Vacances Cubaines » n'offre pas de progression phonétique, ni grammaticale, ni lexicale. C'est pourquoi, il était préférable de construire et introduire une progression dans ces domaines pour que l'assimilation de l'enseignement/ apprentissage suive un rythme d'adaptation équilibré et en accord avec les capacités des apprenants en tant que débutants.

Pour cela, de nombreux documents oraux et transcriptions de ces mêmes documents que comporte "Vacances Cubaines ", n'ont pu être utilisés totalement dans les premières 52 heures, mais se sont intégrés progressivement, à mesure que les apprenants se familiarisaient avec l'enseignement/apprentissage et sentaient plus de souplesse et de flexibilité face à la langue.

Dans ce cas, il faut trouver et sélectionner des documents où la difficulté du contenu linguistique était présentée de manière progressive surtout par rapport au travail des structures grammaticales et morphosyntaxiques.

Il faut également adapter un travail progressif sur la prononciation en privilégiant les sons qui contiennent une difficulté particulière pour les hispanophones. Et finalement, ajouter l'étude du vocabulaire spécifique et regrouper les éléments lexicaux dont les guides se serviraient pour communiquer dans leur pratique et situation professionnelle. 


\section{Construction d'un cours}

\section{Référentiel théorique}

Au lendemain de la deuxième guerre mondiale, les différentes théories en didactique, ont privilégié la notion de communication. Les modèles linguistiques de référence tels que la linguistique structurale et distributionnelle, se sont basés sur des modèles statistiques et distributionnels pour étudier la langue, en prenant compte des mots, des catégories de mots, des emplois syntaxiques c'est à dire, une distribution qui tenait compte de la totalité des environnements.

Cependant, Petar Guberina considérait que, la communication de l'ensemble, n'y était pas interprétée. On y décrivait des modèles où l'homme était absent et cette structure n'était pas adaptée à la langue en vue d'une communication interpersonnelle.

Dans la méthode structuro-globale audio-visuelle basée sur les recherches de Guberina (SGAV), on a privilégié l'idée que le langage est pluridimensionnel et que l'apprenant apprendra mieux, si l'ensemble de ses sens est soumis à une activité conjointe de ceux-ci.

Dans les méthodes audio-orales le processus d'acquisition s'expliquait comme un ensemble d'habitudes, automatismes linguistiques et réflexes de façon spontanée, sans passer par la réflexion. Dans la méthode SGAV, on attribuait une valeur à la langue parlée, à partir d'une réflexion.

Pourtant, toutes deux reposaient sur une conception formelle de la langue, où les structures linguistiques, le lexique et la prononciation, influencée, par les théories venant de la psychologie béhavioriste ou gestaliste, étaient prioritaires, c'est à dire où l'organisation formelle de la langue avait plus d'importance que la fonction de signification et expression du langage.

Parallèlement et en réaction à cette conception formelle, à partir d'une préoccupation linguistique et d'une posture du point de vue de la philosophie du langage, apparaît alors dans les années 70 un 36 
renouveau linguistique, à travers l'approche fonctionnelle. La loi de juillet 1971 sur la formation professionnelle des adultes dirige et oriente les besoins de formation en français. L'approche fonctionnelle vient modifier la didactique des langues et questionner le problème de la construction du sens dans l'interaction sociale, de l'intention de signifier dans l'acte du langage. Comme le dit Claude Springer, les travaux de M.A.K. Halliday ont inspiré la méthodologie fonctionnelle lui attribuant un caractère situationnel et sociologique. L'élément linguistique doit être considéré comme faisant partie d'un tout, où l'élément sémantique et l'élément social y sont inclus. L'acte de langage est alors inclus dans l'activité sociale. Ce sont les conditions d'emploi de la langue, dans diverses situations de communication auxquelles on donne priorité, en tenant compte des intentions, c'est à dire des fonctions langagières.

L'approche fonctionnelle a également été influencée par les travaux de Dell Hymes en ethnographie, en ce qui concerne l'acquisition d'une compétence de communication, qui se base sur l'idée qu'il existe " des règles d'emploi régulant la production et interprétation des énoncés appropriés à la situation dans laquelle ils sont produits $»{ }^{4}$. Elle inclut, des facteurs cognitifs, affectifs et sociaux, notion que Canale et Swain, et Sophie Moirand, ont fait évoluer et ont redéfini, en proposant un modèle de description des composantes d'une compétence de communication (linguistique, discursive, référentielle et socioculturelle) qui nous servira de référence. Il est donc nécessaire d'intégrer en même temps, activité langagière et contexte social.

Selon Claude Springer, l'approche fonctionnelle retient également un élément qui est celui des actes de parole définis par Austin et Searle comme " dire c'est faire » c'est à dire ce que l'on fait quand on parle, qui devient fondamental et qui avec la prise en compte des fonctions du langage, vont se transformer en inventaires qui figureront à coté de ceux de la grammaire et du lexique.

\Sophie Moirand, Enseigner à communiquer en langue étrangère (Paris : Hachette, 1982) 15. 
Mais cet enseignement fonctionnel des objectifs langagiers s'accompagne de la prise en considération de l'apprenant et ses besoins et les moyens que l'on va se donner pour l'aider à communiquer. Par conséquent, l'approche fonctionnelle tient compte des besoins langagiers et non langagiers qui détermineront le contenu de la formation à partir d'une étude du public, des situations de communication en fonction de la situation réelle d'enseignement.

Ramener donc cette approche à notre réalité d'enseignement/ apprentissage semblait nécessaire.

Le français fonctionnel se différentie d'un français plus général par le fait que les apprenants vont apprendre « $d u$ français pour en faire un usage déterminé dans des contextes spécifiques ${ }^{5}$.

Les principes retenus par cette approche, pouvaient répondre à la demande d'un public de guides touristiques car on y retrouvait la nécessité de prendre en considération non pas seulement la composante linguistique pour que les apprenants soient capables d'utiliser des modèles phonétiques, grammaticaux, et lexicaux propres, correspondant à leur domaine professionnel, mais également et indissociablement, prendre en considération « l'apprenant, ses besoins, ses motivations, ses stratégies d'apprentissage, etc. ». S'agissant d'un domaine où les premiers contacts avec les francophones sont essentiellement oraux, il était évident que l'importance de la dimension sociale serait l'une des priorités. En effet, cet aspect a été très important car l'appropriation des éléments socioculturels par l'apprenant lui a permis de développer des stratégies lui facilitant la compréhension de l'autre culture en adoptant une attitude d'observation et compréhension des codes sociaux afin de différencier les deux cultures concernées dans une situation interculturelle et faciliter ainsi la communication. Les usages variant d'une culture à l'autre, dans les pratiques sociales par exemple, une sensibilisation constante sur cet aspect était alors nécessaire.

\footnotetext{
${ }^{5}$ Lehmann, 115.
} 
Il était également nécessaire de prendre en considération dans l'enseignement/apprentissage les composantes d'une compétence de communication présentées par S. Moirand car la méconnaissance des différents types de discours par exemple, présents dans les activités professionnelles des guides touristiques, pourraient entraver une conversation entre un guide et un touriste.

Cerner les besoins du public dans une progression cohérente, travailler simultanément les quatre composantes sachant que les frontières qui existent entre l'une et l'autre, ne sont pas toujours bien définies, créer des documents authentiques écrits et surtout oraux en ce qui concerne le français du tourisme pour débutants et trouver des situations de communications qui se rapprochent le plus possible de la réalité concernée, ne paraissait pas évident, surtout si le facteur temps, est constamment présent dans l'enseignement d'un français fonctionnel.

\section{Référentiel méthodologique}

L'éclectisme a de plus en plus d'adeptes dans l'enseignement apprentissage du FLE. Ceci s'explique par son caractère flexible par rapport aux objectifs poursuivis, par les moyens employés et activités proposées. Aujourd'hui, face à une crise des méthodologies due au fait qu'elles ne rendent pas compte des différentes variables présentes dans une situation d'enseignement/apprentissage, constat que font de nombreux auteurs, il semble y avoir une tendance générale à l'éclectisme, que caractérise très clairement Janine Courtillon ${ }^{6}$ :

« L'indéterminisme actuel doit pouvoir tolérer un éclectisme consistant en un ensemble de pratiques ouvertes, négociables et négociées, dirigées vers un but préalablement défini, mais contrôlées, ajustées en fonction de leur efficacité et donc finalement, validées par les résultats qu'elles permettent d'obtenir. »

\footnotetext{
6 Janine Courtillon, "L'unité didactique ", Le Français dans le Monde/Recherches et applications, Méthodes et Méthodologies, 276 (1995) 109-120.
} 
C'est dans cette direction que nous avons dirigé notre travail. Nous avons constamment réfléchi sur les paramètres de la situation d'enseignement et c'est en fonction de cette situation que nous avons essayé d'adapter un programme que nous avons complété, modifié ou corrigé dans le parcours.

Il s'agissait tout d'abord de faire un inventaire des situations de communication afin d'avoir un répertoire de celles-ci pour découvrir dans quelles situations les apprenants allaient devoir ou vouloir apprendre le français. C'est à dire que nous avons cherché les groupes réunissant des formes que les apprenants allaient utiliser dans les échanges qui semblaient les plus effectifs.

Ensuite nous avons cherché à savoir quels étaient les échanges langagiers nécessaires à la situation de communication, relevant de l'expérience professionnelle communicative des apprenants.

Puis, nous avons essayé de déterminer à partir d'une négociation et découverte réciproque des besoins et objectifs, quels éléments langagiers et non-langagiers allaient être nécessaires dans chaque situation tout en sachant que ceux-ci étaient indissociables.

Le schéma que présente Janine Courtillon et qui décrit « les conditions qui permettent un trajet cognitif » à partir de la compréhension pour aller vers la production de la langue, paraissait correspondre de manière adéquate à l'enseignement/apprentissage que nous avons envisagé. Janine Courtillon propose un travail sur :

- des activités de compréhension impliquant reconnaissance et inférence

- des activités de mémorisation impliquant reproduction et remémoration (phonétique/lexicale/morpho-syntaxique)

- des activités de production impliquant rappel de phrases mémorisées et création de phrases nouvelles.

A partir de ce schéma, nous avons pu " greffer » et ajouter des activités qui s'adaptaient au contexte dans lequel nous avons travaillé et selon les attentes et réactions des apprenants en particulier. 
S'agissant de débutants, d'apprenants avec des habitudes et fonctionnements cognitifs différents, ceci exigeait un enseignement porté sur la variabilité et la souplesse de la méthodologie à utiliser. De cette manière, on pouvait puiser dans diverses méthodes communicatives qui pouvaient servir de fil conducteur, tout en travaillant les objectifs spécifiques de manière progressive, introduisant les éléments les plus adaptables de "Vacances Cubaines». Ces éléments concernaient surtout les aspects socioculturels, sa dimension interculturelle et ceux de l'auto-apprentissage car ils sont généralement présentés en langue maternelle, mais surtout parce qu'ils représentaient une formation continue qui aiderait les à gérer leur apprentissage et élargir leurs connaissances, en dehors de la salle classe. Il a fallu également tenir compte des objectifs présentés dans les modules et des tâches que les apprenants devaient réaliser en les ramenant à l'utilisation de structures linguistiques plus simples.

Outre l'élément de compréhension et production, il était indispensable que se crée dans la classe une ambiance de confiance où les sentiments de crainte, de honte ou peur du ridicule se dissipent, pour faire place à la motivation et au désir de parler. C'est en travaillant sur des activités ludiques par exemple, que l'on a pu briser la glace et en même temps, travailler sur les aspects psychologiques qui surviennent lorsqu'on apprend une langue étrangère.

Un autre aspect, et bien sûr tout aussi important que les autres, est celui du lexique. Le lexique est "constitutif du domaine» et « maîtriser un domaine, c'est maîtriser les mots qui y circulent »?

C'est à partir d' un travail collectif et d' une exploration mutuelle enseignant/apprenant, que l'on a pu extraire le vocabulaire correspondant aux contenus langagiers dans les situations professionnelles. A partir de l'analyse des types d'échanges langagiers et des situations que les apprenants rencontraient dans l'exercice de leurs activités professionnelles, nous avons travaillons sur une

\footnotetext{
${ }^{7}$ Gisèle Kahn, « Différentes approches pour l'enseignement du français sur objectifs spécifiques », Le Français dans le Monde/Recherches et applications, Méthodes et Méthodologies, 276 (1995) 144-152.
} 
sélection lexicale en accord avec les besoins et objectifs des apprenants. C'étaient les guides eux-mêmes qui pouvaient apporter, en langue maternelle, les connaissances du vocabulaire nécessaire à leur activité professionnelle, et pouvaient découvrir et faire découvrir à l'enseignant, à l'aide d'échantillons de discours spécialisés recueillis, leurs besoins en ce qui concerne le lexique et champs lexicaux à travailler.

Un dernier aspect indispensable à tout enseignement et au travail envisagé dans l'espace même de sa construction, a été celui de l'évaluation. Cet élément devait être constant et présent à tout moment. Nous avons fait une évaluation qui portait sur les quatre habiletés de communication en insistant sur l' expression et compréhension orales, en nous centrant sur les intérêts des apprenants et ses résultats. Étant donné que les guides touristiques se retrouvaient majoritairement en situation professionnelle orale, nous étions plus portés à évaluer la compétence de production à partir de productions spontanées en situation de communication orale. Si nous allions surtout évaluer les aspects de la compréhension et expression orales, nous avons tenu compte de la capacité à utiliser des éléments tels que la compétence phonétique, linguistique et prosodique, la morphosyntaxe, la variété et pertinence du lexique, la variété et cohérence des registres.

Nous avons intégré une évaluation fondée sur la capacité à faire certains actes essentiels et de manière acceptable et ayant conscience de la volonté de dire, de la part de l'apprenant et non de l'erreur linguistique en tant que norme.

L'évaluation pouvait être formative, réalisée au moyen d'une observation quotidienne et d'un questionnaire en fin de session, qui permettrait en même temps, à l'enseignant, de réfléchir sur son rôle de médiateur, animateur ou conseiller et sur les aspects socio-affectifs des apprenants.

\section{Détermination du contenu}

L'inventaire des situations de communication fait antérieurement, au moment de la définition des besoins, correspondant 
aux situations professionnelles de notre public, a représenté la base qui a servi à déterminer les contenus langagiers et culturels du cours. Ces situations de communication ne représentaient pas, toutefois, la totalité des contenus car certains d'entre eux sont venus, au cours de l'apprentissage, se greffer selon les besoins. Ces situations étaient tout de même, le fil conducteur qui nous a permis d'établir et répartir ces contenus dans un enchaînement conversationnel, tenant compte de la progression des apprenants, en accord avec les priorités que nous avons considéré nécessaires et pertinentes.

Cette progression était répartie par des ensembles organisés de tâches communicatives à accomplir selon les situations de communication déjà accordées et mises en relation avec les objectifs et activités que propose "Vacances Cubaines » en les adaptant concrètement au contexte de la classe. Chaque objectif était un module qui contenait des activités différentes selon la compétence de communication à travailler et dans une progression des techniques pour apprendre, en commençant par la compréhension orale pour aller vers l'expression orale.

Chaque module comportait donc :

- une partie en espagnol qui se référait à l'aspect socioculturel " Nous informer et informer les autres »

- une deuxième partie d'activités de compréhension orale respectant une progression qui comportait un travail de préparation "Se préparer ", un travail d'entraînement "S'entraîner » et un travail de production « Mettre en pratique "

- une troisième partie d'activités d'expression orale comportant un travail progressif de préparation, entraînement et production

- une quatrième partie en majorité en espagnol qui se réfère à un travail sur l'auto-apprentissage à partir de «Conseils pour apprendre ». 
A ceci, on a ajouté l'étude des aspects suivants :

- un travail progressif de prononciation et phonétique adapté aux hispanophones

- un contenu lexical nécessaire et en rapport avec les situations de communications mentionnées auparavant

- un contenu grammatical suivant une progression selon les situations de communications mentionnées auparavant

Le choix des matériaux d'enseignement tenait compte des besoins des apprenants, des ressources dont nous disposions, des discours produits dans les situations données et de la réaction des apprenants face à l'apprentissage. Puisque nous privilégié les tâches communicatives orales, nous avons utilisé les enregistrements oraux de "Vacances Cubaines » mais également ceux des méthodes communicatives existantes, proposant des enregistrements fabriqués dont le contenu linguistique était plus simple pour un début d'apprentissage. Le fait d'avoir recours aux documents fabriqués ne représente pas d'inconvénients quant à l'authenticité recherchée qui peut être créée au sein des interactions et déclenchée par ces mêmes documents.

Un autre type de document auquel on a eu recours a été la vidéo, à travers laquelle une riche exploitation interculturelle a pu se réaliser et motiver l'apprentissage des apprenants.

Les documents écrits utilisés étaient de différents types. En ce qui concerne la composante culturelle, ils étaient en majorité en langue maternelle pour faciliter l'introduction de cette composante et initier les apprenants à l'auto-apprentissage.

D'autres ont été choisis selon leur contenu linguistique, thématique, communicatif et culturel tels que les brochures ou dépliants en français se trouvant à notre portée pour les mettre en rapport avec l'image que les apprenants avaient de leurs capacités communicatives. Afin d'affirmer les structures grammaticales abordées, nous avons utilisé différents exercices grammaticaux puisés dans les différents livres de grammaire à notre disposition. 
Ces activités étaient travaillées selon la chronologie des situations rencontrées dans la réalité professionnelle et les différentes habiletés à faire acquérir dans un processus qui partait la compréhension orale à l'expression orale pour produire et enfin créer des échanges communicatifs en tenant compte des caractéristiques interactionnelles.

L'une des activités à laquelle nous avons donné beaucoup d'importance était celle de la mise en situation au moyen du jeu de rôle et de simulations faisant ainsi appel à des opérations linguistiques et cognitives diverses afin que les apprenants évaluent eux-mêmes leur compétence de communication et pour évaluer constamment leur progression. D'autres se basaient sur le travail en groupe et des techniques de créativité pour permettre la préparation et élaboration d'un travail sous forme de petits exposés correspondant à leurs différentes activités professionnelles.

Ces activités étaient accompagnées d'exercices oraux de repérage et de discrimination, en phonétique par exemple, des exercices de narration et description permettant de travailler l'identification et repérage dans le temps et l'espace, ainsi que des aspects de la qualification et caractérisation en incluant des types de discours rendant compte des variétés de registres et différences socioculturelles. Les exercices écrits étaient de type lacunaire et de recomposition permettant à l' apprenant de classer et mettre en relation des éléments d'un dialogue par exemple.

Par rapport à la répartition des contenus, elle se faisait avec souplesse et flexibilité en proposant une organisation des tâches à réaliser, permettant à nos apprenants, qui n'étaient pas habitués à gérer leur apprentissage, d'initier un travail sur cet aspect, même si cette répartition n'était pas définitive.

Cette répartition était surtout faite à partir de situations qui proposaient des évènements de communication en suivant une chronologie proche et représentative des situations rencontrées dans la réalité professionnelle des guides touristiques pouvant se combiner 
à une analyse linguistique qui partait du descriptif pour aller vers l'argumentatif. Cependant, les guides touristiques dans leurs activités professionnelles se trouvant face à face avec les touristes, devaient se présenter, décrire, caractériser, conseiller, puis alerter, expliquer, répondre à des questions et dans cette progression, ils étaient en permanence face au besoin d'argumenter et être prêts à réagir face aux situations imprévisibles qui pouvaient se présenter à tout moment. Il était donc important, de tenir compte de ces situations imprévisibles et d'introduire dans la progression, des modules ou activités qui contenaient ces situations de communication.

A des moments précis du parcours d'apprentissage nous avons prévu une évaluation orale au moyen d'exposés oraux qui étaient en rapport avec les pratiques professionnelles des guides touristiques. Par exemple, ils présentaient à la fin de chaque session, un exposé qui contenait la présentation des caractéristiques d'un parc national en ajoutant une description générale de la faune et la flore caractéristique du parc choisi et en permettant aux apprenants observateurs de participer à travers des questions et évaluer également la compréhension orale. A partir de cette détermination des contenus, et sachant qu'ils pouvaient être modifiés à tout moment, quatre modules ont été élaborés en vue de cette formation de 52 heures pour guides touristiques débutants en considérant une progression basée sur un circuit touristique qui commence à l'arrivée au Costa Rica et se termine dans un parc national.

\section{MODULE 1 :}

\section{«Premiers contacts »}

Ce module représente le premier travail à réaliser avec les apprenants et le premier contact qui se fait entre les deux cultures et les deux langues. Il s'agit surtout de sensibiliser les apprenants aux différences culturelles qui existent entre les deux pays et de donner 
une première approche linguistique tout en initiant une prise de conscience de l'importance des stratégies d'apprentissage, de leur auto-apprentissage, et des premières réactions psycho-affectives qui, naturellement, surgissent lorsqu'on commence l'apprentissage d'une langue étrangère. Il s'agit également de commencer à parler de soimême et réaffirmer le « je » dans la langue cible. Ce module contient également les premiers éléments linguistiques dans lequel on donne priorité à la phonétique et prononciation de sons qui n'existent pas en espagnol afin que les apprenants se familiarisent progressivement avec ces nouveaux sons.

\section{Nous informer et informer les autres :}

- Comparer les idées sur ce qu'est un bon guide et l'idée que les Français se font sur eux

- Se sensibiliser par rapport aux différences entre les habitudes costariciennes et les habitudes françaises quant au tutoiement entre touristes et professionnels

\section{Apprendre à communiquer}

\section{Compréhension orale :}

Se préparer

- Se sensibiliser avec des mots français et reconnaître la langue parmi d'autres

- Se sensibiliser avec des expressions utiles pour donner la bienvenue à des touristes

- Se sensibiliser avec des expressions utiles pour se présenter devant un groupe de touristes

- Se sensibiliser avec des expressions utiles pour présenter un collègue (le chauffeur du car par exemple)

- Se sensibiliser avec des expressions utiles pour exprimer ses goûts et ses préférences 
S'exercer

- S'entraîner pour localiser des expressions utiles dans un dialogue de bienvenue à des touristes

- $\quad$ S'entraîner pour localiser des expressions utiles pour se présenter en tant que guide, devant un groupe de touristes

- $\quad$ S'entraîner pour localiser des expressions utiles pour présenter un collègue (le chauffeur du car par exemple)

- S'entraîner pour localiser des expressions utiles pour exprimer ses goûts et ses préférences

Mettre en pratique

- Reconnaître lorsqu'on est en train de donner la bienvenue

- Reconnaître lorsqu'un guide se présente

- Reconnaître lorsqu'un guide présente un collègue

- Reconnaître ce qu'une personne aime ou pas, ce qu'elle préfère ou pas

\section{Expression orale :}

Se préparer

- Découvrir comment nous pouvons donner la bienvenue à un groupe de touristes

- Découvrir comment se présenter devant un groupe de touristes

- Découvrir comment présenter un collègue devant un groupe de touristes

- Découvrir comment parler de ses goûts et préférences

S'exercer

- S'entraîner pour donner la bienvenue

- $\quad$ S'entraîner pour se présenter

- S'entraîner pour présenter un collègue

- $\quad$ S'entraîner dans la prononciation de noms français au moment de faire l'appel

- S'entraîner pour exprimer ses goûts et préférences

Mettre en pratique

- Simuler donner la bienvenue à un groupe de touristes 
- Simuler se présenter devant un groupe de touristes

- Simuler présenter un collègue devant un groupe de touristes

- Simuler faire l'appel des noms de touristes français

- Simuler des conversations en exprimant ses goûts et préférences

\section{Conseils pour apprendre :}

- Se sensibiliser par rapport aux différences culturelles

- Se sensibiliser par rapport aux différences entre le « Tu » et le «Vous"

- Comment mémoriser ce que nous voulons retenir?

\section{Phonétique :}

- S'entraîner dans la prononciation de noms français au moment de faire l'appel

- $\quad$ Travailler le son [y] en opposition aux sons [i], [u]

- $\quad$ L'opposition [œ] [yn]

- $\quad$ L'opposition des sons [s] [z]

\section{Vocabulaire :}

- Expressions de salutations

- Les professions

- Les nationalités

- Les expressions : avoir + faim, soif, sommeil, envie de, besoin de

- Las expressions utiles para exprimer goûts et préférences (j'aime, je préfère, je ne supporte pas, j'ai horreur de, c'est pas mal, etc.)

\section{Contenu grammatical :}

- $\quad$ Les verbes Avoir et Être

- Il est + adjectif ou profession / C'est + article

- $\quad$ Les verbes du $1^{\text {er }}$ groupe (-er)

- Le verbe S'appeler / Faire / Connaître 


\section{- $\quad$ Articles définis, indéfinis, contractés \\ - $\quad$ Le genre et le nombre \\ - La négation}

\section{MODULE 2 :}

\section{«Informer les touristes sur un programme touristique avant d'initier un circuit »}

Ce deuxième module consiste à introduire des informations linguistiques et culturelles par rapport à la présentation d'un programme touristique. Il s'agit de travailler pour se préparer à répondre aux curiosités que les touristes peuvent avoir au sujet de la présentation d'un programme touristique et éclaircir les doutes en ce qui concerne les activités qu'ils vont réaliser dans les temps accordés. Ce module tient compte également de l'importance pour le guide de pouvoir donner des explications qui lui permettent de se situer, d'aider le touriste à se situer, ainsi que situer un objet et un lieu dans l'espace et le temps. En effet, le guide doit non seulement dire quelle sera la destination du tour, mais tenir compte des situations accidentelles qui peuvent se présenter et se préparer à les comprendre et à répondre à ces situations (un touriste perdu, qui demande une adresse, une direction).

\section{Nous informer et informer les autres :}

- Se sensibiliser quant aux attentes des français par rapport aux salutations : se serrer la main par exemple

\section{Apprendre à communiquer}

\section{Compréhension orale :}

Se préparer

- Se sensibiliser avec des structures utiles pour présenter un programme de visite touristique 
- Se sensibiliser avec les expressions utiles d'un touriste qui explique ce qu'il pense faire dans la journée

- Se sensibiliser avec des expressions utiles pour situer un objet ou un lieu et comment y arriver

- Se sensibiliser avec les questions que posent les touristes par rapport au programme touristique

- Se sensibiliser avec les questions que posent les touristes pour se situer dans un lieu et savoir comment y arriver

S'exercer

- S'entraîner pour reconnaître des structures utiles pour présenter un programme de visite touristique

- S' S' entraîner pour reconnaître les expressions utiles d'un touriste qui explique ce qu'il pense faire dans la journée

- S'entraîner pour reconnaître des expressions utiles pour situer un objet ou un lieu et les explications pour y arriver

- S'entraîner pour reconnaître les questions que posent les touristes par rapport au programme touristique

- S'entraîner pour reconnaître les questions que posent les touristes pour se situer dans un lieu et savoir comment y arriver

\section{Expression orale :}

Se préparer

- Découvrir comment nous pouvons présenter un programme

- Découvrir les expressions d'un touriste qui explique ce qu'il pense faire dans la journée

- Découvrir les expressions utiles pour situer un objet ou un lieu et comment y arriver

- Découvrir les questions que posent les touristes par rapport au programme touristique

- Découvrir les questions que posent les touristes pour se situer dans un lieu et savoir comment y arriver

S'exercer

- S'entraîner pour présenter un programme devant un groupe de touristes 
- S'entraîner pour expliquer ce qu'on pense faire dans la journée

- S'entraîner pour situer un objet ou un lieu et comment y arriver

- S' $\quad$ 'entraîner pour répondre aux questions que posent les touristes par rapport à un programme touristique

- $\quad$ S'entraîner pour répondre aux questions que posent les touristes pour situer un lieu et savoir comment y arriver

\section{Mettre en pratique}

- Simuler la présentation d'un programme touristique devant un groupe de touristes

- Simuler des conversations où les touristes expliquent ce qu' ils pensent faire dans la journée

- Simuler situer un objet ou un lieu et expliquer comment y arriver

- Simuler interroger et répondre aux questions que posent les touristes par rapport à un programme touristique

- Simuler interroger et répondre aux questions que posent les touristes pour situer un lieu et savoir comment y arriver

\section{Conseils pour apprendre :}

- Comment s'adapter aux clients et ses habitudes?

\section{Phonétique :}

- Travailler les oppositions

/E/ /Ø/

[b] [v]

- Travailler l'intonation : interrogation/affirmation

\section{Vocabulaire :}

- Noms de lieux touristiques

- Objets faisant partie des services proposés (dans un bus touristique)

- vocabulaire de lieux naturels qu'on trouve au Costa Rica

- Les nombres

- L'heure 


\section{Contenu grammatical :}

- Le verbe Aller

- Les prépositions : à, en, $a u, a u x+$ noms des villes et pays

- $\quad$ Les prépositions de lieux : dans, sur, sous, à coté de...

- $\quad$ Le futur Proche

- Le pronom ON

- Les verbes irréguliers : vouloir/pouvoir/prendre/dormir

- L'interrogation

\section{MODULE 3 :}

\section{«Informer le touriste sur les précautions à prendre au cours de leur séjour dans le pays »}

Le troisième module est centré sur un travail qui veut répondre aux besoins des guides de prévenir les touristes sur les dangers auxquels ils pourraient être exposés en considérant les différences culturelles existantes entre les deux pays. Les touristes ne sont pas habitués à certaines pratiques culturelles costariciennes comme le type d'alimentation (surtout les aliments que l'on vend dans la rue et l'eau à laquelle ils ne sont pas habitués), le climat (le soleil, les moustiques), le comportement des conducteurs et piétons (le respect du code de la route et les précautions que le piéton doit prendre), la sécurité (les voleurs).

Cependant, pour ne pas alarmer, brusquer et faire peur aux touristes face à la présentation de ces dangers, l'explication de ces précautions doit être faite de manière à faire comprendre aux touristes qu'ils sont exposés à ces dangers dans toutes les capitales du monde et que ce ne sont pas des problèmes caractéristiques du Costa Rica mais qu'il s'agit d'être conscient face à certains changements dans leurs habitudes, éviter ainsi des complications lors de leur séjour dans le pays et ne pas présenter une vision trop négative du Costa Rica.

Au niveau linguistique, les structures nécessaires à cet objectif se basent sur l'expression de la prévention (faire attention à), de la nécessité (il est nécessaire de, il faut) et du conseil (je vous conseille de). 


\section{Nous informer et informer les autres :}

- S'informer sur les préférences des français quant aux vacances

\section{Apprendre à communiquer}

\section{Compréhension orale :}

Se préparer

- Observer les expressions utiles pour alerter un touriste

- Observer les expressions utiles pour donner des conseils à un touriste

- Observer les expressions utiles pour donner des instructions sur les précautions à prendre dans le pays par rapport à l'alimentation, aux vols, au climat

- Observer les expressions utiles pour parler du climat S'exercer

- Reconnaître les expressions utiles para alerter un touriste

- Reconnaître les expressions utiles pour donner des conseils à un touriste

- Reconnaître les expressions utiles pour donner des instructions sur les précautions à prendre dans le pays par rapport à l'alimentation, aux vols, au climat

- Reconnaître les expressions utiles pour parler du climat

\section{Expression orale :}

Se préparer

- Se sensibiliser avec les expressions utiles pour alerter un touriste

- Se sensibiliser avec les expressions utiles pour donner des conseils à un touriste

- Se sensibiliser avec les expressions utiles pour donner des instructions sur les précautions à prendre dans le pays par rapport à l'alimentation, aux vols, au climat

- Se sensibiliser avec les expressions utiles pour parler du climat 
S'exercer

- $\quad$ S'entrainer pour utiliser les expressions utiles pour alerter un touriste

- S'entraîner pour utiliser les expressions utiles pour donner des conseils à un touriste

- S'entraîner pour utiliser les expressions utiles pour donner des instructions sur les précautions à prendre dans le pays par rapport à l'alimentation, aux vols, au climat

- S'entraîner pour utiliser les expressions utiles pour parler du climat

Mettre en pratique (soltarse)

- Simuler des conversations pour alerter un touriste

- Simuler des conversations pour donner des conseils à un touriste

- Simuler des conversations pour donner des instructions sur les précautions à prendre dans le pays par rapport à l'alimentation, aux vols, au climat

- Simuler des conversations pour parler du climat

Conseils pour apprendre :

- Comment améliorer par soi-même son expression orale ?

\section{Phonétique :}

- Travailler les oppositions :

[õ] [on]

[ã] [an]

[3] [j]

- Discrimination féminin/masculin

\section{Vocabulaire :}

- Révision des nombres et de l'heure

- Les vêtements

- Les affaires personnelles

- L'alimentation

- Les expressions pour parler du climat (il fait beau, mauvais/il pleut/la chaleur/le soleil/'humidité...) 


\section{Contenu grammatical :}

Prévenir, conseiller, recommander, expression de la nécessité

- $\quad$ Faire attention à + nom

- Il faut + infinitif / Il ne faut pas

- Devoir + infinitif / Vous ne devez pas

- Je vous conseille de / je vous recommande de

- L'impératif (affirmatif/négatif)

- Les adjectifs possessifs

- $\quad$ Les verbes irréguliers : Mettre, Boire, Pleuvoir

\section{MODULE 4 :}

\section{« Présenter et décrire un parc national costaricien »}

Le quatrième et dernier module correspond à la présentation et description d'un parc national costaricien. Sachant que chaque parc présente des particularités différentes et qu'il nous est impossible de les étudier dans leur totalité, ce module se base surtout sur un travail en groupe, nous permettant d'accéder aux caractéristiques variées des principaux parcs nationaux costariciens. Ce module comporte un travail de caractérisation et description tout d'abord générale des parcs nationaux (le situer géographiquement, parler du type de climat, végétation et animaux principaux qu'on y trouve) et progressivement, une étude de plus en plus spécifique des principales particularités de la flore et la faune de chaque parc étudié.

Au moment de la visite d'un parc national, le guide doit se présenter, présenter le programme, expliquer les précautions que les touristes doivent prendre, et ce qui est permis de faire ou ne pas faire (fumer, parler à haute voix durant l'observation de la faune, ne pas toucher, faire attention à, etc.).

On retrouve donc implicitement, regroupés dans ce module, le travail réalisé auparavant dans les trois modules précédents permettant ainsi une reprise des contenus qui peut représenter un premier rappel 
et évaluation des objectifs des modules 1,2 et 3, ainsi qu'une nouvelle étude de caractérisation et description. Une fois le travail de ce module terminé, et en guise de conclusion d'une première étape de cette formation, on peut envisager une simulation qui reprendrait les objectifs principaux des quatre modules parcourus.

\section{Nous informer et informer les autres :}

- Comparer ce qu'on peut ou ne pas faire au moment d'accueillir un groupe de touristes

\section{Apprendre à communiquer}

\section{Compréhension orale :}

S'exercer

- S'entraîner à la compréhension de différents types de visites

- S' entraîner à la localisation des expressions utiles pour décrire et caractériser la faune et la flore dans un parc national

- S'entraîner à donner des explications sur un parc national

\section{Expression orale :}

Se préparer

- Découvrir le lexique et les expressions utiles pour faire une description d'un parc national costaricien

- Découvrir les moyens linguistiques utilisés pour caractériser la faune et la flore d'un parc national costaricien

- Réfléchir sur ce qui se dit lorsqu'on conduit un groupe à une visite guidée dans un parc national

S'exercer

- Utiliser le lexique et les expressions utiles pour faire une description d'un parc national costaricien

- Utiliser les moyens linguistiques pour caractériser la faune et la flore d'un parc national costaricien

- S'entraîner pour présenter et décrire un parc national, sa faune et sa flore 


\section{Mettre en pratique}

- Simuler présenter et décrire un parc national costaricien

- Simuler une explication et une description de la flore et la faune d'un parc national costaricien

\section{Conseils pour apprendre :}

- Quelle place occupe la grammaire dans l'apprentissage de l'expression orale?

\section{Phonétique :}

- Travailler les oppositions :

[ẽ] [õ]

[ẽ] [ã]

[jẽ] [jen]

\section{Vocabulaire :}

- $\quad$ Les couleurs et les formes

- Les 5 sens et le vocabulaire respectif

- Noms d'animaux et de végétation caractéristiques d'un parc national costaricien

\section{Contenu grammatical :}

- Les adjectifs qualificatifs

- Le féminin des adjectifs

- Les comparatifs

- Quelques marqueurs spatiaux

- Quelques marqueurs temporels dans le discours (pour commencer, d'abord, ensuite, en fin...)

- Quelques marqueurs de la transformation (se transformer, devenir, se changer en...)

Au terme de cette formation, notre analyse nous a tout d'abord amenés à nous pencher sur les différents paramètres situationnels 
nécessaires à la mise en place de la formation en question. Pour cela, nous avons contacté les institutions concernées et obtenu de leur part, sans grandes difficultés, le financement.

La caractérisation du public et ses besoins s'est définie dans la construction de la formation et s'est découverte progressivement à mesure que l'enseignement/apprentissage prenait forme. Par rapport à la demande et besoins des apprenants, un des aspects important à corriger parce que nous ne l'avons pas pris en considération, ni inclut dans notre enseignement/apprentissage, était celui de la notion même de francophonie qui ne devrait pas se limiter uniquement à la culture française mais élargir l'étude à d'autres cultures telle que la canadienne car sa population est représentative dans le domaine du tourisme au Costa Rica.

En dernier lieu, nous avons construit un cours basé sur l'approche fonctionnelle qui nous a permis de centrer l'enseignement/ apprentissage sur l' idée que l'acte de langage est inclus dans l'activité sociale et les facteurs cognitifs, affectifs et socioculturels ont été et sont le pivot autour duquel doit se construire ce type de formation.

Sur cette base théorique, la méthodologie proposée par les auteurs de "Vacances Cubaines " imbibée d'une dimension sociale nous a donné les moyens de construire quatre modules localisés et adaptés au contexte costaricien en y apportant les modifications nécessaires à tout enseignement/apprentissage spécialisé, qui corresponde à la réalité économique, sociale et institutionnelle du Costa Rica et plus concrètement encore, à la réalité dans l'espace et le temps de notre public dans notre classe.

Aujourd'hui, de plus en plus de projets et demandes se concrétisent par rapport à la création de nouvelles formations dans le domaine du français du tourisme, qui ne se limitent pas à la réalité costaricienne, mais s'étendent à la région centraméricaine. Par conséquent, il est nécessaire de continuer à former des formateurs qui forment les professionnels au niveau régional afin de répondre comme il se doit aux besoins et demandes. 
Finalement, même si on se base sur une théorie, si on élabore une méthodologie et des objectifs à travers l'organisation d' un contenu d'enseignement/apprentissage, faisant partie d'une dialectique entre théorie et pratique inhérente et enrichissante en ce qui concerne la théorisation de la pratique, il se crée dans « le temps et l'espace » de la classe, tout un monde du domaine linguistique, socioculturel et affectif, qui modifie constamment d'une manière ou d'une autre, le contenu des modules élaborés, qui ne peut y être reflétée de manière constante et transparente, mais qui est là, présent à tout moment, et que seuls, les apprenants et l'enseignant, découvrons dans l'espace même de la construction de la formation que nous avons envisagée. 